\title{
Unusual Perirenal Metastasis from Lung Adenocarcinoma
}

\author{
Akciğer Adenokarsinomunun Nadir Perirenal Metastazı
}

\section{Işıl Başara, Canan Altay, Mustafa Seçil}

Dokuz Eylül University Faculty of Medicine, Department of Radiology, İzmir, Turkey

\section{Introduction}

A 70-year-old man underwent left upper lobectomy for lung adenocarcinoma in 2012. At the time of operation, there was no sign of systemic metastasis. After surgery, chemotherapy and radiotherapy regimens were applied for treatment. In the $2^{\text {nd }}$ year following surgery, the patient presented with weakness, weight loss, cough and bilateral flank pain. He underwent thoracic and abdominal computed tomography (CT) examination. CT examination revealed ipsilateral and contralateral recurrent lung tumours, hepatic and splenic metastases (Figure 1). In both perirenal regions, especially in the posterior parts, there were heterogeneity, enhanced strands, linear and millimetric nodular densities (Figure 2, 3). This finding was interpreted as an evidence of perirenal metastasis. A new chemotherapy regimen was planned for treatment.

Retroperitoneum is divided into three components in the region of kidneys by anterior perirenal fascia (Gerota's fascia), posterior perirenal fascia (Zuckerkandl's fascia). These two fasciae connect each other laterally in order to form lateroconal fascia. The three components are the anterior pararenal space, posterior pararenal space and the perirenal space (1). The perirenal space leans between the renal capsule and the anterior and posterior renal fasciae. This space contains plenty of fat (2).
The perirenal region is commonly involved in a large variety of neoplastic and non-neoplastic pathologies. The adrenal glands and the kidneys are common metastatic sites. However, isolated perirenal metastasis is unusual. Metastases from lung cancer and some other cancers, such as malignant melanoma, prostate and breast carcinoma may be detected (3).

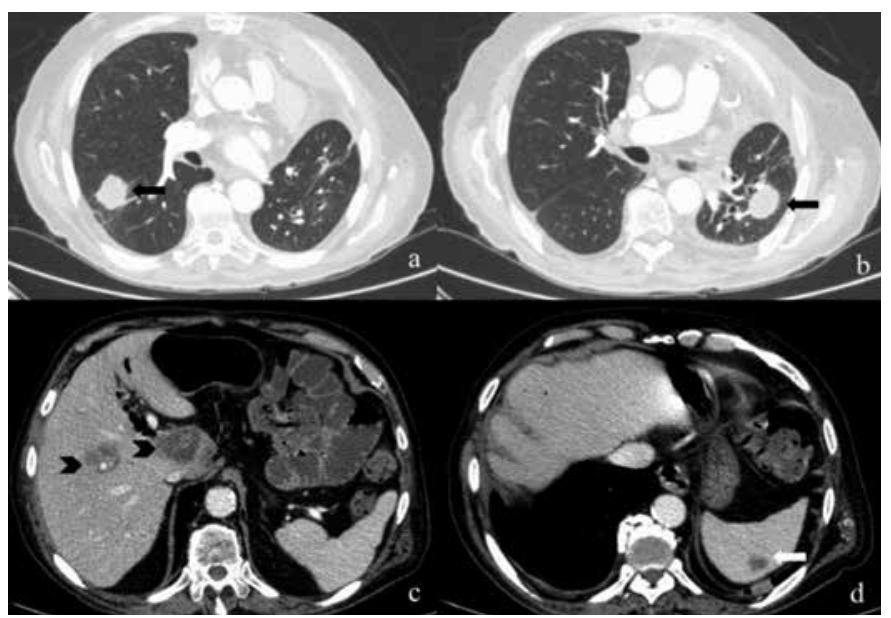

Figure 1. a, b) Lung computed tomography (CT) examination, there are recurrent masses at bilateral lower lobes of lung (black arrows), c, d) Abdominal computed tomography (CT) examination, there are hypodense nodular liver (arrow heads) and splenic (white arrow) metastases

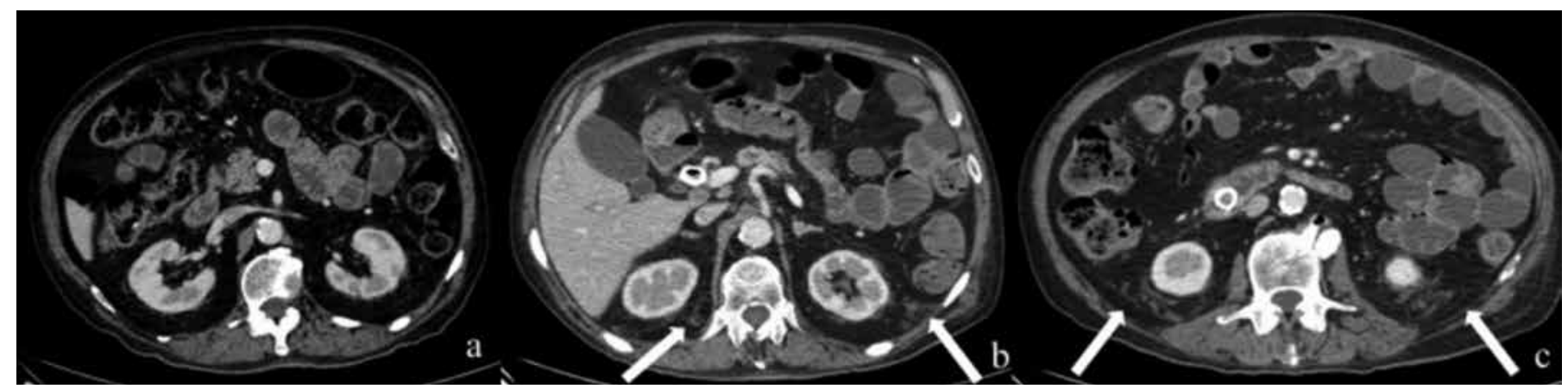

Figure 2. Axial abdominal computed tomography (CT) images, a) At the time of diagnosis, there is no lesion in perirenal sites, b, c) There are heterogeneity, strands, linear and milimetric nodular densities in perirenal region compatible with metastasis and infiltration of lung carcinoma (arrows) 


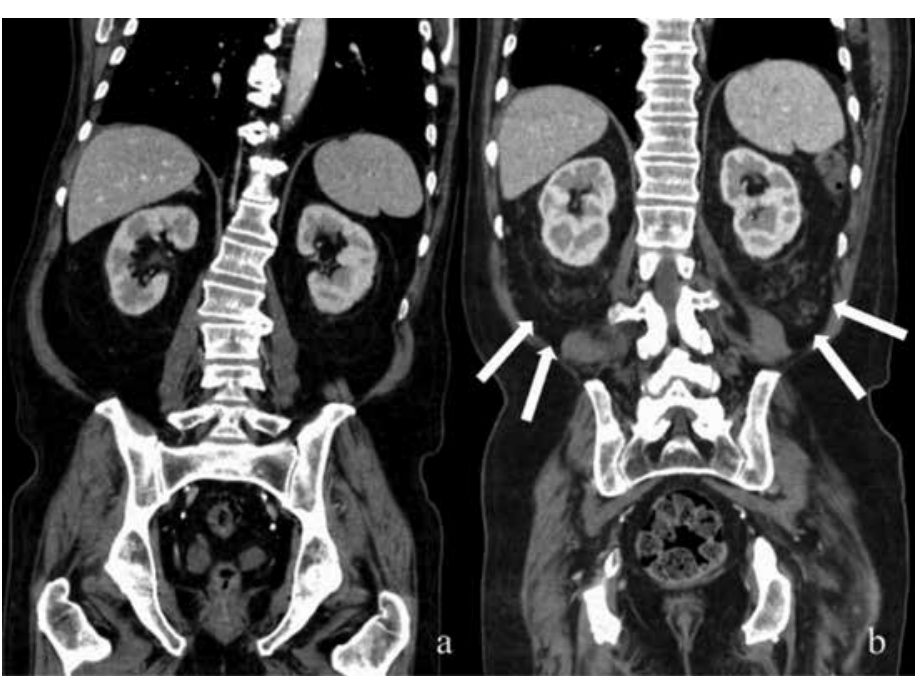

Figure 3. Coronal abdominal computed tomography (CT) images, a) At the time of diagnosis, perirenal region is normal, b) Bilateral perirenal metastatic infiltration is seen (arrows)

Metastatic spread through the lymphatics is thought to be the mechanism of perirenal metastasis $(3,4)$. The lymphatic system in the perirenal region communicates with mediastinal lymph nodes through the thoracic duct and diaphragmatic perforations as well as with para-aortic lymph nodes through the renal hilum. In addition, haematogenous spread may cause metastasis (5).

There are only six cases of perirenal metastasis from lung carcinomas reported in the English literature $(1,2,3,4)$. In five cases, there were perirenal nodules or masses as metastasis $(2,3,4)$. Only in one case, diffuse metastatic perirenal fat infiltration was detected. CT findings in this case were similar to that in our case. As in our case, there were linear heterogeneous densities at the perirenal site (1). To the best our knowledge, our case is the second case of diffuse perirenal fat tissue infiltration.

In the differential diagnosis of perirenal heterogeneity, linear and millimetric nodular densities and enhancement secondary to perinephric fatty tissue infection acute/subacute retroperitoneal haemorrhage, perirenal lymphoma and liposarcoma can be thought (1).

Perirenal metastasis presenting with perirenal heterogeneity and strands are very rare. In cases of primary lung carcinoma, diffuse stranding of perinephric region metastatic involvement should be kept in mind in the differential diagnosis.

Key Words: Lung carcinoma, metastasis, perirenal

Anahtar Kelimeler: Akciğer kanseri, metastaz, perirenal

\section{References}

1. D'Souza DL, Heinze SB, Dowling RJ, Unusual presentation of perirenal lung metastases. Australas Radiol 2006;50:246-248.

2. Shirkhoda A. Computed tomography of perirenal metastases. J Comput Assist Tomogr 1986;10:435-438.

3. Wilbur AC, Turk JN, Capek V. Perirenal metastases from lung cancer: CT diagnosis. J Comput Assist Tomogr 1992;16:589-591.

4. Kwak TI, Kim DS, Kim JJ, Yoon DK, Cho JH, Koh SK. Lung cancer metastasizing to ipsilateral renal cell carcinoma and contralateral perirenal space. BJU Int 1999;83:512-513.

5. Bechtold RE, Dyer RB, Zagoria RJ, Chen MY. The perirenal space: relationship of pathologic alprocesses to normal retroperitoneal anatomy. Radiographics 1996;16:841-854. 